\title{
Single-molecule imaging reveals the oligomeric state of functional TNF $\alpha$ - induced plasma membrane TNFR1 clusters in cells
}

Article

Accepted Version

Karathanasis, C., Medler, J., Fricke, F., Smith, S., Malkusch, S., Widera, D., Fulda, S., Wajant, H., van Wijk, S. J. L., Dikic, I. and Heilemann, M. (2020) Single-molecule imaging reveals the oligomeric state of functional TNF $\alpha$-induced plasma membrane TNFR1 clusters in cells. Science Signaling, 13 (614). eaax5647. ISSN 1937-9145 doi:

https://doi.org/10.1126/scisignal.aax5647 Available at https://centaur.reading.ac.uk/88348/

It is advisable to refer to the publisher's version if you intend to cite from the work. See Guidance on citing.

To link to this article DOI: http://dx.doi.org/10.1126/scisignal.aax5647

Publisher: AAAS

All outputs in CentAUR are protected by Intellectual Property Rights law, including copyright law. Copyright and IPR is retained by the creators or other copyright holders. Terms and conditions for use of this material are defined in the End User Agreement. 


\section{www.reading.ac.uk/centaur}

\section{CentAUR}

Central Archive at the University of Reading

Reading's research outputs online 
One-sentence summary: Ligand binding triggers the oligomerization of TNFR1 monomers and dimers into trimers and higher-order oligomers.

\section{Editor's summary:}

\section{Oligomerizing for optimal activity}

Drugs that prevent the activation of the TNF $\alpha$ receptor TNFR1 are of great interest because of the many roles of this ligand/receptor pair in pathophysiological processes such as inflammation. Karathanasis et al. determined the oligomerization state of TNFR1 by quantitatively analyzing single-molecule superresolution microscopy data. TNF $\alpha$ stimulation triggered the clustering of TNFR1 monomers and dimers into trimers and nonamers. Forms of TNFR1 with mutations that impaired basal dimerization or that abolished ligand binding did not assemble into higher-order oligomers upon TNF $\alpha$ stimulation. These results provide insight into the oligomerization states of TNFR1 that must be targeted before and after TNF $\alpha$ stimulation.

\section{Single-molecule imaging reveals the oligomeric state of functional TNFa-induced plasma membrane TNFR1 clusters in cells}

Christos Karathanasis ${ }^{1}$, Juliane Medler ${ }^{2}$, Franziska Fricke ${ }^{1}$, Sonja Smith ${ }^{3}$, Sebastian

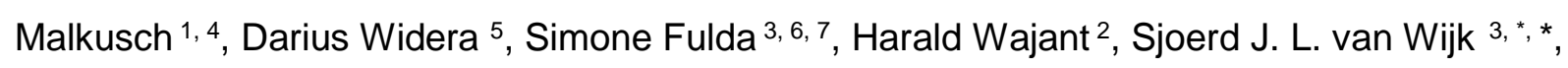
Ivan Dikic ${ }^{8,}{ }^{*}$, Mike Heilemann ${ }^{1,{ }^{*}}$

${ }^{1}$ Institute of Physical and Theoretical Chemistry, Goethe University, Max-von-Laue-Strasse 7, 60438 Frankfurt am Main, Germany

2 Division of Molecular Internal Medicine, Department of Internal Medicine II, University Hospital Würzburg, Auverahaus, Grombühlstrasse 12, 97080 Würzburg, Germany

${ }^{3}$ Institute for Experimental Cancer Research in Paediatrics, Goethe University, Komturstrasse 3a, 60528 Frankfurt am Main, Germany

${ }^{4}$ Current address: Institute of Clinical Pharmacology, University Hospital of Frankfurt, GoetheUniversity, Theodor-Stern-Kai 7, 60590 Frankfurt am Main, Germany ${ }^{5}$ Stem Cell Biology and Regenerative Medicine Group, School of Pharmacy, University of Reading, RG6 6UB, Reading, United Kingdom 
${ }^{6}$ German Cancer Consortium (DKTK), Heidelberg, Germany

${ }^{7}$ German Cancer Research Centre (DKFZ), Heidelberg, Germany

${ }^{8}$ Institute of Biochemistry II, Goethe University Medical School, Theodor-Stern-Kai 7, 60590 Frankfurt am Main, Germany and Buchmann Institute for Molecular Life Sciences (BMLS), Goethe University, Max-von-Laue-Strasse 15, 60438 Frankfurt am Main, Germany

*Corresponding author. Email: s.wijk@kinderkrebsstiftung-frankfurt.de (S.J.L.v.W.); ivan.dikic@biochem2.de (I.D.); heilemann@chemie.uni-frankfurt.de (M.H.) 


\begin{abstract}
Ligand-induced tumor necrosis factor receptor 1 (TNFR1) activation controls NF-KB (nuclear factor kappa-light-chain-enhancer of activated B-cells) signaling, cell proliferation, programmed cell death, and survival, and is crucially involved in inflammation, autoimmune disorders, and cancer progression. Despite the relevance of TNFR1 clustering for signaling, oligomerization of ligand-free and ligand-activated TNFR1 remains controversial. At present, models range from ligand-independent receptor pre-dimerization to ligand-induced oligomerization. Here, we used quantitative, single-molecule superresolution microscopy to study TNFR1 assembly directly in native cellular settings and at physiological cell surface abundance. In the absence of its ligand TNFa, TNFR1 assembled into monomeric and dimeric receptor units. Upon binding of TNFa, TNFR1 clustered predominantly into trimers but also into higher-order oligomers. A functional mutation in the pre-ligand assembly domain (PLAD) of TNFR1 resulted in only monomeric TNFR1, which exhibited impaired ligand binding. In contrast, a form of TNFR1 with a mutation in the ligand-binding CRD2 subdomain retained the monomer-to-dimer ratio of the unliganded wildtype TNFR1 but exhibited no ligand binding. These results underscore the importance of ligand-independent TNFR1 dimerization in NF-kB signaling.
\end{abstract}

\title{
Introduction
}

The tumor necrosis factor (TNFa) receptor superfamily (TNFRSF) consists of 29 receptors and can be divided into TNFa receptor-associated factor (TRAF)-interacting receptors, death receptors (DRs), and decoy receptors, which capture ligands, but do not stimulate intracellular signaling pathways, thereby counterbalancing other receptors (1-3). DRs contain intracellular death domains (DD) that control cell proliferation, inflammation, pro-survival signaling through NF-kB) as well as programmed forms of cell death (4). TNFR1 is a prototypical, 55-kD type 1 transmembrane DR (5). Signaling occurs through indirect recruitment of TNFR1-associated death domain protein (TRADD), TRAF2, and the TRAF2-interacting E3 ubiquitin ligases cellular inhibitor of apoptosis proteins-1 and -2 (clAP1/2), resulting in activation of pro-survival NF-KB responses (5), which are involved in cell proliferation (6) and inflammation (7). Furthermore, when IAP activities are compromised, TNFR1 activation may lead to the induction of apoptosis or, when caspase-8 is inhibited, possibly necroptosis (8) (9). In summary, TNFR1 controls cell survival and programmed cell death pathways (10) and is involved in vital processes, such as hematopoiesis (11) and protection from bacterial infections (12). Disturbances in TNFa-TNFR1 signaling underlie inflammation (10), autoimmune diseases (13), and cancer (14). 
The intracellular DD of TNFR1 is connected through a flexible transmembrane region to four extracellular cysteine-rich domains (CRD1 to CRD4) that each contain six cysteine residues by which three disulfide bridges are composed (15). Binding of TNFa to TNFR1 leads to TNFaTNFR1 interactions in which TNFR1 molecules bind into the clefts formed between the three protomers of a trimer of TNFa. X-ray crystallography studies of several TNFRSF ligands alone or in complex with the ectodomain of their receptors suggest the formation of trimeric TNFa:TNFR1 complexes (16). This finding is consistent with the requirement of clustering of TNFRSF members into larger assemblies for efficient signaling, because the trimeric scaffold of TNFa can accommodate three TNFR1 monomers (15). However, it remains less clear how TNFR1 clustering is organized, achieved, and maintained in unliganded states and upon ligand binding within the native environment, namely the plasma membrane of a mammalian cell.

TNFa interacts with TNFR1 through residues within CRD2 and CRD3 (17). CRD1 is most likely not directly involved in ligand binding but seems to stabilize CRD2 and CRD3 for optimal ligand binding (18). CRD1 is the most distal CRD from the plasma membrane and accommodates the pre-ligand assembly domain (PLAD) (17). The PLAD mediates TNFR1 dimerization even in the absence of ligand, and PLAD-PLAD binding affinities are in the micromolar range (19). Based on this and other evidence, a two-step model of TNFRSF activation has been proposed (20). In the first step, ligand-induced formation of trimeric TNFSF (ligand)-trimeric TNFRSF (receptor) complexes occurs. In the second step, these complexes are thought to form secondary aggregates that are mediated through PLAD-PLAD interactions between trimeric TNFSF-trimeric TNFRSF complexes. How the PLAD mediates ligand-dependent or independent TNFR dimerization, trimerization, or even oligomerization and subsequent TNFR1 signaling, remains unclear.

Thus far, TNFR1 oligomerization has largely been investigated by biochemical receptor crosslinking $(17,21)$ and/or crystallization experiments $(15,22)$ in which TNFRSF ectodomains are isolated from the native membrane environment. Based on these data, different TNFR1 oligomerization models were proposed $(16,17,21,23)$. Although these types of study have generated much valuable information, it remains unclear how TNFR1 distributions are organized in their native environment, the mammalian plasma membrane. Ideally, receptor organization into functional units should be observed directly in physiological cellular settings to maintain the native membrane environment and to avoid the perturbation of protein-protein 
interactions that orchestrate receptor oligomerization. Optical microscopy seems the obvious tool for such investigations, but has been limited in spatial resolution for a long time. Developments in advanced optical microscopy have provided the necessary spatial resolution and sensitivity to determine the oligomeric state of a receptor within a protein assembly (24). Single-molecule localization microscopy (SMLM) is particularly suited to provide quantitative information on protein assembly by exploiting information from a fluorophore-label conjugated or bound to a specific receptor (25). Such information may be the kinetics of photoswitching of the fluorescent reporters $(26,27)$ or the binding kinetics of fluorophore labels $(28)$. For example, quantitative SMLM has revealed a selective organization of Toll-like receptor 4 (TLR4) in response to specific bacterial ligands (29).

Here, we used quantitative $\operatorname{SMLM}(24,25)$ in combination with total internal reflection fluorescence (TIRF) illumination to interrogate how TNFR1 assembles into functional units or clusters on the plasma membrane of a mammalian cell. Specifically, we studied unliganded and TNFa-activated TNFR1, together with functional mutants of TNFR1. For this purpose, we reconstituted TNFR1/2 double knock-out $\left(^{-/}\right)$mouse embryonic fibroblasts (MEFs) with TNFR1-mEos2 to recapitulate complete cellular TNFR1 functionality. Furthermore, we explored the relevance of the PLAD in controlling TNFR1 receptor oligomeric clustering and NF-kB signaling.

\section{Results}

\section{A cellular system to image and quantify TNFR1 clustering using superresolution microscopy}

To address how TNFR1 distributions are organized in the plasma membrane, we generated a genetic system that enabled quantitative analysis of TNFR1 oligomerization status in the plasma membrane of intact cells using superresolution microscopy. For this, TNFR1/2 double knockout (-/-) mouse embryonic fibroblasts (MEFs) were transduced with lentivirus to stably express full-length human TNFR1, which was fused at the $\mathrm{C}$ terminus to the photoactivatable fluorescent protein mEos2 (TNFR1/2/- + TNFR1-mEos2). mEos2 is suitable for superresolution PALM imaging (30), and enables the calculation of molecule numbers because of its photoblinking properties $(26,27,29,31)$. Previously, we have shown that TNFR1-mEos2 fusion proteins do not undergo mEos2-induced dimerization (32). The absence of TNFR2 in the reconstituted TNFR1/2/- TNFR1-mEos2 MEFs enabled analysis without confounding factors of TNFR1 clustering and signaling, because it has been reported that in certain 
scenarios TNFR2 modulates TNFR1 activation (20). Western blotting analysis of total cell lysates with anti-TNFR1 antibody revealed the existence of a major band corresponding to the molecular mass of TNFR1-mEos2 and several non-specific background bands (Fig. 1A). The total amounts of TNFR1 in the reconstituted TNFR1/2- + TNFR1-mEos2 MEFs was relatively increased compared to TNFR1 levels of the human cervix carcinoma HeLa cell line. The antiTNFR1 antibody used here for detection of TNFR1 levels recognizes only human TNFR1 and does not cross-react with mouse TNFR1. Furthermore, apart from the major TNFR1 isoform 1 , this antibody also recognizes additional, smaller TNFR1 isoforms. However, these additional bands are most likely not involved in ligand binding and TNFR1 clustering, because Flagtagged TNFa immunoprecipitation of TNFR1 from HeLa cells and TNFR1-mEos2 from TNFR1/2/ + TNFR1-mEos MEFs only enrich the major TNFR1 bands and not the these smaller isoforms (Fig. S1).

TNFR1/2/- TNFR1-mEos2 MEFs exhibited normal TNFa-induced NF-kB signaling, as revealed by examining TNFa-induced IKBaphosphorylation and subsequent degradation patterns, which were similar to those of wild-type (WT) MEFs (upon 3 hours serum-starvation) (Fig. 1B). In contrast and as expected, TNFR1/2/- MEFs and TNFR1/2-- MEFs expressing mEos2 alone were defective in TNFa-induced NF-kB signaling. The NF-kB signaling status of the $T N F R 1 / 2^{--}$+ TNFR1-mEos2 MEFs was further confirmed by immunofluorescence microscopy analysis of TNFa-induced p65 nuclear accumulation. Indeed, upon stimulation with TNFa, TNFR1/2/- + TNFR1-mEos2 MEFs were similar to TNFa-treated WT MEFs in terms of the extent of nuclear accumulation of p65, confirming that reconstitution of $T N F R 1 / 2^{-\alpha} \mathrm{MEFs}$ with TNFR1-mEos2 did not interfere with signal progression (Fig. 1C). In contrast, TNFR1/2-MEFs did not show nuclear p65 accumulation in response to TNFa (Fig. 1C).

These observations suggest that stable reintroduction of TNFR1-mEos2 into TNFR1/2- MEFs functionally restored TNFa-induced and TNFR1-mediated NF-KB signaling. Furthermore, although TNFR1 abundance was greater in TNFR1/2- + TNFR1-mEos2 MEFs compared to HeLa cells, TNFR1 did not display autoactivation in the absence of TNFa, suggesting functional restoration of TNFR1-mediated NF-KB signaling to near-endogenous settings (Fig. 1B, 1C).

We assessed whether reconstitution of TNFR1/2-/ MEFs with TNFR1-mEos2 also restored TNFa-induced, TNFR1-mediated programmed cell death.Together, these results confirm the 
functionality of the reconstituted TNFR1-mEos2 fusion protein in TNFR1/2 ${ }^{-1}+$ TNFR1-mEos2 MEFs by rescue of TNFR1-dependent, TNFa-mediated signaling events during short- and long-term episodes of ligand exposure.

\section{Quantitative PALM imaging of TNFR1 multimerization in the mammalian plasma membrane}

To determine the membrane distribution status of TNFR1 in reconstituted TNFR1/2- + TNFR1mEos2 MEFs, cells were subjected to 24 hours of serum-starvation to reset TNF $\alpha$-TNFR1 signaling and PALM imaging to visualize the distribution of single TNFR1-mEos2 clusters with sub-diffraction spatial resolution (Fig. 2, A and B). These images were further analyzed to determine the number of mEos2 proteins per cluster (and hence, the number of TNFR1-mEos2 within a cluster), following published procedures $(26,27,31)$ that were previously used to determine the oligomeric state of Toll-like receptor 4 (29) (Fig. S3A, S3B, S3C and S3D).

Quantitative analysis of serum-starved TNFR1-mEos2 at the plasma membrane of TNFR1/2-+ TNFR1-mEos2 MEFs revealed a density of $1.4+/-0.4$ TNFR1-mEos 2 clusters $/ \mu m^{2}$ (Table 1) and an organization of TNFR1-mEos2 into $66+/-4 \%$ monomeric and $34+/-4 \%$ dimeric TNFR1 fractions (Fig. 2C, Table 2 and Table S1). Notably, on the surface of serum-starved TNFR1/2-- mEos2 MEFs, trimeric or higher-order TNFR1-mEos2 clusters were not detected.

Next, we determined how exogenously added TNFa alters the TNFR1-mEos2 membrane organization and clustering distribution. For this purpose, we used a fully-functional SNAPtagged TNFa (SNAP-Flag-TNC-TNF $\alpha$ ), which had a binding affinity of $6+/-3 \mathrm{ng} / \mathrm{ml}$ in a HeLa cell-based assay, which is on a similar order of the binding affinity of huTNFa-Flag-TNC-GpL with a value of $K_{D}=12+/-5 \mathrm{ng} / \mathrm{ml}$ (Fig. S4A and S4B), and whose functionality was verified by cell survival assays (Fig. S4C). We incubated serum-starved TNFR1/2-- + TNFR1-mEos2 MEFs for 30 min with SNAP-Flag-TNC-TNFa conjugated to Alexa Fluor 647 (TNFa-SNAPAF647) at $4{ }^{\circ} \mathrm{C}$ to prevent TNFR1 internalization upon ligand binding (34). Applying fluorophore-labelled ligand allowed the distinct selection of ligand-bound TNFR1-mEos2 molecules and quantitative analysis. PALM images of TNFR1/2- + TNFR1-mEos2 MEFs treated with TNFa-SNAP-AF647 demonstrated colocalization of a fraction of TNFR1-mEos2 with TNFa-SNAP-AF647 (Fig. 3, A and B). Quantitative analysis revealed a total TNFR1 density of $1.6+/-0.7$ clusters $/ \mu \mathrm{m}^{2}$ (Table 1$)$. 
The blinking histogram (Fig. 3C) was analyzed with a total of 511 different models ranging from one to nine components and covering all possible combinations of monomer to 9-mer (9 models for one component, 36 for two components, 84 for three components, 126 for four components, 126 for five components, 83 for six components, 35 for seven components, 9 for eight components, and one for nine components). The best model was identified by statistical analysis using the Bayesian information criterion.

TNFa-colocalized TNFR1-mEos2 (yellow circles) organized into $13+/-2 \%$ monomers, $64+/$ $2 \%$ trimers, and a fraction of higher-order oligomers that could best be approximated with the distribution function for 9-mers, yielding $23+/-3 \%$ (Fig. 3C, Table 2 and Table S2). In contrast, the ligand-free TNFR1-mEos2 population upon TNFa-SNAP-AF647 incubation was detected in a $41+/-4 \%$ monomer and $59+/-4 \%$ dimer distribution (Fig. 3D, Table 2 and Table S3).

Thus, we could demonstrate that in unliganded TNFR1/2/- TNFR1-mEos2 MEFs, TNFR1mEos2 adopted a monomer/dimer distribution, and that upon ligand binding, TNFR1-mEos2 predominantly organized into trimers and higher order oligomers with only a small fraction of monomers left. Ligand-free monomeric TNFR1-mEos2 also shifted into a dimeric TNFR1mEos2 population upon ligand incubation. These findings indeed support a model of a dynamic equilibrium of monomeric and dimeric TNFR1 in the membrane which redistribute into trimers and higher order oligomers after ligand binding (20).

\section{Quantitative PALM of TNFR1 with mutations in the PLAD or ligand binding site}

To understand the role of the TNFR1 PLAD in TNFR1-mEos2 dimerization, higher-order clustering and global membrane distribution patterns, a K32A point mutation was generated in TNFR1-mEos2 CRD1 which is located in the predicted PLAD of TNFR1 and which abrogates self-assembly (17). Quantifying TNFR1-mEos2 K32A distribution in serum-starved TNFR1/2-+ TNFR1-mEos2 K32A MEFs revealed the presence of only monomeric TNFR1 on the plasma membrane (Fig. 4A, Table 2 and Table S4). We observed only a low amount of specific binding of TNF $\alpha$-SNAP-AF647 to TNFR1/2- + TNFR1-mEos2 K32A MEFs (Fig. 4B and Table S5) $\left(K_{D}=91+/-33 \mathrm{ng} / \mathrm{ml}\right.$ compared to $K_{D}=16+/-3 \mathrm{ng} / \mathrm{ml}$ of WT TNFR1-mEos2 (Fig. S2B)). 
In contrast, the TNFR1 CRD2 mutation N66F, which is located in the ligand binding site, displayed a $54+/-3 \%$ monomeric and $46+/-3 \%$ dimeric distribution in TNFR1/2-- + TNFR1mEos2 N66F MEFs (Fig. 4C, Table 2 and Table S6). As expected, we did not observe any ligand binding to TNFR1/2/- TNFR1-mEos2 N66F MEFs (Fig. 4D and Table S7). These results suggest that a functional PLAD is essential to generate the fraction of ligandindependent dimeric TNFR1 at the mammalian plasma membrane and that TNFa-binding site mutations do not interfere with ligand-independent PLAD-mediated TNFR1 clustering.

However, it must be noted that both TNFR1 K32A and N66F were found at lower amounts in total cell lysates (Fig. 4E) compared to WT TNFR1, which was confirmed by FACS analysis (Fig. S2A). In PALM experiments, only a small number of cells was found with a sufficiently high abundance of TNFR1 for imaging and analysis, which might have led to an experimental bias. Apparently, the K32A and N66F mutations in TNFR1-mEos2 might affect protein stability, localization, or inter-organelle TNFR1 transport, which has been reported for several TNFR1 PLAD mutations observed in TNF Receptor Associated Periodic Syndrome (TRAPS), a rare genetic autoinflammatory disorder (35). It is possible that mutated TNFR1-mEos2 fails to fold properly and therefore can no longer be anchored in the membrane, thereby affecting PALM imaging because this method detects intracellular, membrane-proximal TNFR1-mEos2 (TIRF illumination). TNF binding studies indicate that TNFR1 -mEos2 K32A still binds to ligand, while this binding was lost for TNFR1-mEos2 N66F (Fig. S2B). PALM imaging and quantitative analysis of cells with a sufficient abundance of receptors and exposed to TNF $\alpha$-SNAP-AF647 revealed a monomeric TNFR1 (for the K32A mutant) and a mixture of monomers and dimers (for the N66F mutant) (Fig. 4C, 4D). In addition, both TNFR1-mEos2 K32A and N66F, display suppressed NF- $\mathrm{KB}$ activation measured through I $\mathrm{kB} \alpha$ phosphorylation and degradation by Western blot analysis (Fig. 4F).

\section{Discussion}

Multimeric clustering of TNFR1 is essential for survival and cell fate signaling and mediates activation of the NF-kB pathway. The spatial organization of TNFR1 in ligand-free and ligandbound states in the physiological membrane environment remains, however, largely unclear. Here, we studied TNFR1 distribution into different states of multimerization in unliganded and TNF $\alpha$-treated cells. To monitor the TNFR1 clustering distribution by PALM, we reconstituted TNFR1/2-- MEFs with full-length human TNFR1-mEos2. mEos2 is a 26-kDa photoactivatable 
protein that was fused to the C-terminus of TNFR1. Although the C-terminally located death domain of TNFR1 is essential for triggering TNFR1-mediated NF-KB and cell death signaling, C-terminal fusion of mEos2 did not substantially interfere with TNFR1-mediated functions. Stable expression of TNFR1-mEos2 in TNFR1/2- MEFs completely restored TNF $\alpha$-induced IKB $\alpha$ phosphorylation and degradation as well as the induction of programmed forms of cell death mediated through TNFR1. In sum, these data suggest functional TNFR1 signaling in TNFR1-mEos2 reconstituted TNFR1/2/- MEFs.

We next applied quantitative PALM to determine the molecular distribution of TNFR1-mEos2 in the plasma membrane. Our data revealed that $66 \%$ of TNFR1-mEos 2 was monomeric and $34 \%$ was dimeric in the absence of ligand. These observations suggest that low-affinity TNFR1 PLAD-PLAD interactions primarily mediate the formation of TNFR1 dimers, which are likely to exist in a dynamic equilibrium with monomeric TNFR1 (20). Indeed, this notion is supported by the almost exclusive monomeric distribution of the PLAD mutant TNFR1-mEos2 K32A (Fig. 4A).

Upon ligand binding, most of the TNFR1-mEos2 molecules became organized into trimeric receptor clusters, as well as higher-order clusters that could be extracted using a single additional function for a 9-mer to fit the experimental data of fluorophore blinking (31). Formation of TNFR1 9-mers could potentially be organized by PLAD-PLAD-mediated interactions of ligand-bound TNFR1 trimers.

The affinity of TNFa for the PLAD mutant TNFR1-mEos2 K32A was approximately 5-fold less than that for WT TNFR1-mEos2 (Fig. S2B) but was still high enough to substantially occupy TNFR1-mEos2 K32A at the concentration of $100 \mathrm{ng} / \mu$ l used for functional PALM imaging (Fig. 4B). Nevertheless, TNF $\alpha$ was not only unable to trigger formation of TNFR1 trimers and nonamers, but also completely failed to elicit an NF-kB response. This finding initially suggested that ligand-bound TNFR1 trimers and nonamers are the signaling active species. However, chimeric death receptors, in which the extracellular domain of TNFR1 has been replaced by TNFR2 or CD95, bind to soluble TNFa and CD95L, respectively, but are not activated by these ligands (36). Instead, strong signaling is initiated from these chimeric receptors when oligomerized ligand trimers are used for stimulation. The binding of soluble TNF $\alpha$ and soluble CD95L to three molecules of TNFR2 and CD95, respectively, suggests that 
two or more trimeric TNFa receptor complexes are required for proper receptor engagement. Therefore, it seems likely that liganded nonameric TNFR1 species are the major and dominant signaling active TNFR1 fraction. A careful selection of fitting parameters, together with the evaluation of different possible models through their fitting quality, enabled us to conclude that TNF $\alpha$-activated TNFR1-mEos2 mainly organizes into trimers and likely 9-mers, whereas tetramers or pentamers are less likely to occur in the membrane. Although this does not exclude PLAD-PLAD-mediated interactions of two ligand-bound TNFR1 trimers, we only see a low probability for the presence of 6 -mers as intermediate scaffold.

Although we used a concentration of $100 \mathrm{ng} / \mathrm{ml}$ TNFa, which saturates TNFR1 (Fig. S2B), we detected only about $20+/-9 \%$ TNFR1 clusters bound to TNFa in PALM images. We believe that this is in part due to our imaging and detection window (TIRF illumination), which extends about 100 to $200 \mathrm{~nm}$ deep into the cell. We cannot exclude that we also detected intracellular TNFR1, such that receptor densities determined from PALM images (Table 1) might not only represent TNFR1 on the cell membrane, but also TNFR1 present in the Golgi network, an organelle known to be involved in TNFR1 trafficking and recycling $(37,38)$. The ligand-bound TNFR1 density in TNFR1/2/ - + TNFR1-mEos2 MEFs was $0.3+/-0.1$ clusters/ $/ \mathrm{m}^{2}$. Considering a mean surface of TNFR1/2/- TNFR1-mEos2 cells of $2539+/-608 \mu \mathrm{m}^{2}$, this translates into $762+/-182$ TNFR1-TNFa complexes per cell (Fig. 3A, 3B, Table 1). Considering that there are $13 \%$ monomeric, $64 \%$ trimeric and $23 \%$ nonameric TNFR1 in TNFa-activated cells, this suggests a total number of $3139+/-750$ TNFR1 molecules per cell. These data are consistent with TNFR1 copy numbers per cell as determined by luciferase assay, which reported $2698+/-2518$ copies of TNFR1 (Fig. S2B).

In sum, our data support a model of TNFR1 activation by soluble TNFa in which the PLADPLAD interaction not only facilitates TNFa binding by the formation of dimeric TNFR1 species in the plasma membrane with higher affinity for TNFa, but is also required for clustering of inactive or poorly active liganded TNFR1 trimers into signaling active higher-order clusters (Fig. 5). Our statistical analysis of single-molecule superresolution data indicates that these higher-order clusters are likely to be nonamers. It is tempting to speculate that the strength of the PLAD-based auto-affinity of TNFRSF receptors determines whether clustering to signaling competent receptor nonamers or oligomers occurs spontaneously after binding of soluble ligand trimers due to sufficient PLAD-PLAD auto-affinity (as in the case of TNFR1) or whether this requires stimulation with physically linked soluble ligand trimers (as in the case of TNFR2 or CD95) due to a too low auto-affinity. Our work furthermore demonstrates that advanced 
superresolution microscopy together with quantitative analysis can extract the oligomeric state of unliganded and ligand-bound receptors of the TNFRSF directly in its native environment, the plasma membrane of the cell.

\section{Materials and Methods}

\section{Cell lines, reagents, and antibodies}

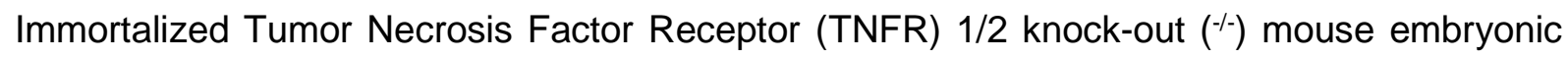
fibroblasts (MEFs) were obtained from Daniela Männel (Regensburg) and maintained in Roswell Park Memorial Institute (RPMI) medium (ThermoScientific), supplemented with $10 \%$ fetal bovine serum (FBS) (Gibco), $1 \%$ Glutamax (Thermo Scientific), $1 \%$ Penicillin/Streptomycin (Life Technologies, Inc.), at $37 \stackrel{\circ}{\circ}$ with $5 \% \mathrm{CO}_{2}$. Human cervical carcinoma cells (HeLa) and human embryonic kidney (HEK) 293T Phoenix-ECO Ecotropic packaging cells were obtained from and authenticated by the American Type Culture Collection (ATCC). HeLa and Phoenix-ECO 293Ts were maintained in Dulbecco's Modified Eagles Medium (DMEM), supplemented with $10 \%$ FBS, $1 \%$ Penicillin/Streptomycin and $1 \%$ sodium pyruvate (Life Technologies, Inc.), at $37^{\circ} \mathrm{C}$ with $5 \% \mathrm{CO}_{2}$. All cell lines were regularly monitored for Mycoplasma infection. Recombinant human TNF $\alpha$ was purchased from Peprotech and Biochrom, BV6 was a kind gift from Domagoj Vucic (Genentech, Inc), and zVAD.fmk was purchased from Bachem. All other chemicals were obtained from Sigma-Aldrich or Carl Roth, unless indicated otherwise. Antibodies used were monoclonal mouse anti-human vinculin (V9131, Sigma), anti-human IkBa (\#9242, Cell Signalling Technology), anti-phosphoIkBa (Ser ${ }^{32}$, 14D4, 2859, Cell Signalling), anti-NF-kB p65 (C-20, sc-372, Santa Cruz Biotechnology), mouse anti-TNFR1 H-5 (sc-8436, Santa Cruz), rabbit anti-TNFR1 C25C1 (\# 3736S, Cell Signalling). We note that anti-TNFR1 C25C1 also targets a truncated isoform of TNFR1 in some cell lines, as reported by the company.

\section{DNA cloning, virus production, and viral transduction}

Template plasmids encoding full-length human TNFR1 (TNFRSF1A, Uniprot P19438) and monomeric Eos2 (mEos2) were described previously (39). The pBABE-puro retroviral vector was obtained from Addgene (plasmid \# 1764). Genetic C-terminally tagged TNFR1-mEos2 fusions were generated using standard cloning PCR, with a 5' Kozak sequence for translation 
initiation and a 3' stop codon and lacking the signal peptide residues $1-29$, using conventional restriction digest cloning. Cloning details and primer sequences are available upon request. Clones were verified with Sanger DNA sequencing. To generate high-titer viral particles, Phoenix-ECO 293T helper cells were seeded at a density of $7.5 \times 10^{5}$ cells per well in six-well plates (Greiner), 24 hours before transfection in DMEM without antibiotics. Transfections with pBABE-puro, pBABE-puro-mEos2 and wild-type, K32A and N66F pBABE-puro-TNFR1mEos2 were performed with FuGENE HD Transfection Reagent (Promega), according to the manufacturer's instructions in serum-reduced OptiMEM (Thermo Scientific). Medium was

replaced with DMEM with serum and antibiotics at 12 hours post-transfection and viral supernatants were collected 48 and 72 hours later. Supernatants were pooled, briefly centrifuged and frozen for storage at $-80^{\circ} \mathrm{C}$. Viral supernatants were thawed, filtered through $0.45-\mu \mathrm{m}$ filter units, and mixed with $10 \mu \mathrm{g} / \mathrm{ml}$ Polybrene to infect $5 \times 10^{5} \mathrm{TNFR} 1 / \mathrm{2}^{-/} \mathrm{MEFs}$ overnight at $37^{\circ} \mathrm{C}$ in complete RPMI. Stably transduced cells were selected in complete RPMI with puromycin $(2 \mu \mathrm{g} / \mathrm{ml})$. Single clones were isolated and evaluated for TNFR1 expression by Western blotting.

\section{Cell lysis and Western blotting}

Cells were washed twice with ice-cold Phosphate-Buffered Saline (PBS) (Gibco) and lysed in lysis buffer $(30 \mathrm{mM}$ Tris- $\mathrm{HCl} \mathrm{pH}$ 7.4, $150 \mathrm{mM} \mathrm{NaCl}, 1 \%(\mathrm{v} / \mathrm{v})$ Triton X-100 and $10 \%(\mathrm{v} / \mathrm{v})$ glycerol), supplemented with Complete Protease Inhibitors (Roche). Lysates were incubated on ice for $20 \mathrm{~min}$. and centrifuged at $16.060 \mathrm{~g}$ at $4 \stackrel{\circ}{\circ} \mathrm{C}$ for $20 \mathrm{~min}$. Cleared cell lysates were boiled in 2 x Laemmli Sample Buffer (4\% (w/v) SDS, $20 \%$ (v/v) glycerol, 120 mM Tris-HCI pH $6.8,0.02 \%$ bromophenol blue) and resolved on SDS-PAGE followed by immunoblotting and detection with goat anti-mouse IgG or goat anti-Rabbit IgG conjugated to horseradish peroxidase (HRP) (Santa Cruz) and enhanced chemiluminescence (Amersham).

\section{Cytokine stimulation}

The indicated stably reconstituted TNFR1/2/- MEFs, WT MEFs and HeLa cells were seeded in six-well plates (Greiner) at a density of $7.5 \times 10^{5}$ cells per well 24 hours prior to stimulation in RPMI with FBS and antibiotics. Cells were serum-starved in RPMI without FBS for 3 hours at $37^{\circ} \mathrm{C}$ and subjected to a single-dose of human recombinant TNF $\alpha$ in serum-free RPMI at the indicated concentrations for the indicated time points at $37^{\circ} \mathrm{C}$. After cytokine stimulation, medium was removed and cells were processed for further analysis. 


\section{Immunofluorescence}

WT, TNFR1/2-- and stably reconstituted TNFR1-mEos2 TNFR1/2-- MEFs were seeded on sterile cover slips in six-well dishes (Greiner) at a density of $7.5 \times 10^{5}$ cells per well 24 prior to stimulation in RPMI with FBS and antibiotics. Cells were serum-starved in RPMI without FBS for 3 hours at $37^{\circ} \mathrm{C}$ and subjected to a single-dose of human recombinant TNFain serum-free $\mathrm{RPMI}$ at the indicated concentrations for the indicated times at $37^{\circ} \mathrm{C}$. Medium was removed and the cells were washed with PBS and fixed in $4 \%$ paraformaldehyde (Santa Cruz) in PBS for $20 \mathrm{~min}$ at room temperature. Cells were washed three times with PBS and permeabilized with $0.2 \%$ Triton-X100 in PBS for 5 min at room temperature. Cells were washed again three times with PBS and blocked with $5 \%$ bovine serum albumin (BSA, Roth) in PBS overnight at $4{ }^{\circ} \mathrm{C}$. Cells were incubated with anti-p65 antibody diluted in $5 \%$ BSA in PBS for 4 hours at room temperature. Cells were subsequently washed three times with $0.1 \%$ Tween-20 in PBS and incubated with Alexa Fluor 647-labeled secondary antibodies in $5 \%$ BSA/PBS. Cells were washed with $5 \%$ BSA/PBS three times, nuclei were counterstained with DAPI (Molecular Probes) and samples were mounted in aqueous mounting medium (ProLong Antifade Gold, Thermo Fischer Scientific) on macroscopic glass supports. Images were acquired on a Leica SP8 confocal laser-scanning microscope (Leica).

\section{Determination of cell death}

Cell lines were seeded at a density of $0.6 \times 10^{4}$ cells / well in sterile 96-well plate (Greiner) in complete medium 24 hours prior to cell death induction. Cells were pretreated with $10 \mu \mathrm{M}$ BV6 alone (to induce extrinsic apoptosis) or combined with $20 \mu \mathrm{M} z V A D$.fmk (to induce necroptosis) for 1 hour at $37^{\circ} \mathrm{C}$. Human recombinant TNF $\alpha(10 \mathrm{ng} / \mathrm{ml})$ was then added and the cells were incubated for 24 hours at $37^{\circ} \mathrm{C}$. Cell death was measured by fluorescence-based microscope quantification of propidium iodide (PI) uptake using Hoechst 33342 and PI double staining (Sigma-Aldrich) using the ImageXpress Micro XLS Widefield High-Content Analysis System and MetaXpress software according to the manufacturer's instructions (Molecular Devices Sunnyvale).

\section{Fluorescence-activated cell sorting analysis}


To analyze TNFR1 cell surface expression, $5 \times 10^{5}$ of the indicated MEFs were washed twice with PBS and incubated for $30 \mathrm{~min}$. at $4{ }^{\circ} \mathrm{C}$ with $\alpha$ TNFR1-PE (\#FAB225P; R\&D) and corresponding mlgG1-PE (\#IC002P; R\&D) isotype control. To remove unbound antibodies, cells were washed again twice with PBS and analyzed with FACS-Calibur (BD Bioscience), following standard procedures.

\section{Cellular binding studies}

Binding affinities of huTNF $\alpha-F-T N C-G p L$ to TNFR1 were determined by cellular equilibrium binding studies. Therefore, aliquots of the indicated cells $\left(4 \times 10^{5}\right)$ were challenged with increasing concentrations of the TNF $\alpha$-GpL-fusion protein (to measure total binding). Values for nonspecific binding were derived for HeLa cells by blocking TNFR1 with $20 \mu \mathrm{g} / \mathrm{ml}$ recombinant human TNF $\alpha$ and for MEFs with TNFR1-deficient MEFs. After $1.5 \mathrm{~h}$ at $37{ }^{\circ} \mathrm{C}$, unbound TNF $\alpha$-GpL-fusion protein was removed by washing the cells three times with ice cold PBS. Finally, cells were resuspended in $50 \mu$ RPMI 1640 media supplemented with $0.5 \%$ FCS and transferred to a black 96-well plate. GpL-activity was measured with the Gaussia luciferase assay kit (New England Biolabs $\mathrm{GmbH}$ ) and the LUmo Luminometer (Anthos Labtec Instruments). Specific binding values were calculated by subtracting the unspecific binding from total binding. To analyze the cellular binding affinity of SNAP-F-TNC-TNF $\alpha$ to TNFR1, homologous competition experiments were performed with aliquots of $4 \times 10^{5} \mathrm{HeLa}$ cells incubated at $37^{\circ} \mathrm{C}$ simultaneously with increasing concentrations of SNAP-F-TNC-TNFaand a constant amount of huTNF $\alpha-F-T N C-G p L(2.5 \mathrm{ng} / \mathrm{ml})$. After 1.5 hours, the cells were washed three times with ice-cold PBS, and GpL-activity was measured as described earlier. Binding curves were analyzed by fitting the data with a Hil1 function implemented in OriginPro 2019 (Equation 1).

$$
f(x)=\operatorname{Int}(0)+(\operatorname{Int}(\operatorname{sat})-\operatorname{Int}(0)) \cdot \frac{c^{n}}{\left(K_{D}^{n}+c^{n}\right)} \quad(\text { Equation } 1)
$$

$\operatorname{Int}(0)$ and Int (sat) indicate the lowest and largest values of measured intensity, respectively; $c$ is the corresponding concentration of TNF $\alpha ; n$ is the number of cooperative sites; and $K_{D}^{n}$ is the dissociation constant. To define the number of huTNF $\alpha$-F-TNC-GpL binding sites per cell $\left(N_{B S}\right)$ and thus the number of TNFR1 molecules per cell, the luciferase activity of a defined amount of huTNF $\alpha$-F-TNC-GpL molecules was measured to determine the luciferase activity per GpL-domain $\left(A_{G p L-d o m a i n}\right)$. Additionally, the maximal specific binding $\left(B_{\max }\right)$ of the corresponding cellular binding studies were obtained by fitting the data with the one-site specific binding function of GraphPad Prism 5. Together with the counted cell numbers $(C M)$, the number of TNFR1 molecules was calculated with equation 2. 


$$
N_{B S}=\frac{\left(B_{\text {max }} \cdot A_{G P L-\text { domain }}\right)}{C N} \quad(\text { Equation } 2)
$$

\section{Cross-linking of FLAG M2 antibody to Protein G Dynabeads}

To crosslink the FLAG M2 antibody to magnetic beads, $25 \mu$ Protein G Dynabead slurry (ThermoFisher) was washed three times with $0.05 \%$ Tween-20/PBS, then incubated with $4 \mu \mathrm{g}$ FLAG M2 monoclonal antibody (Sigma) for $2 \mathrm{~h}$ at $4{ }^{\circ} \mathrm{C}$. Beads were washed in $0.2 \mathrm{M}$ triethanolamine (TAE) in PBS, incubated with $20 \mathrm{mM}$ dimethyl pimelimidate (DMP; Sigma) in $0.2 \mathrm{M}$ TAE pH 8.2 for $30 \mathrm{~min}$ at room temperature, and quenched with $50 \mathrm{mM}$ Tris- $\mathrm{HCl} \mathrm{pH} 7.5$ and three washes with $0.05 \%$ Tween-20/PBS.

\section{FLAG-TNF $\alpha$ immunoprecipitation of TNFR1}

The indicated cell lines were seeded in sterile $10-\mathrm{cm}^{2}$ dishes in complete medium at a density of $1.0 \times 10^{6}$ cells/plate. After $24 \mathrm{~h}$, cells were serum-starved for $24 \mathrm{~h}$ and incubated with FLAGtagged TNF $\alpha$ (Enzo Life Sciences) at $1 \mu \mathrm{g} / \mathrm{ml}$ at $4^{\circ} \mathrm{C}$ in serum-free medium for $30 \mathrm{~min}$. Cells were washed twice with ice-cold PBS and lysed in lysis buffer (30 mM Tris-HCl pH 7.4, 150 $\mathrm{mM} \mathrm{NaCl}, 1 \%(\mathrm{v} / \mathrm{v})$ Triton $\mathrm{X}-100$ and $10 \%(\mathrm{v} / \mathrm{v})$ glycerol), supplemented with Complete Protease Inhibitors (Roche). Lysates were incubated on ice for $20 \mathrm{~min}$. and centrifuged at $16.060 \mathrm{~g}$ at $4{ }^{\circ} \mathrm{C}$ for $20 \mathrm{~min}$. Cleared lysates were incubated with DMP-cross-linked Protein $\mathrm{G}$ magnetic Dynabeads for $24 \mathrm{~h}$ at $4{ }^{\circ} \mathrm{C}$ and washed three times with lysis buffer. Immunoprecipitates were boiled in 2 x Laemmli Sample Buffer (4 \% (w/v) SDS, $20 \%$ (v/v) glycerol, $120 \mathrm{mM}$ Tris-HCl pH 6.8, $0.02 \%$ bromophenolblue) and resolved by SDS-PAGE. Immunoprecipitated proteins were detected by immunoblotting with the appropriate antibodies and goat anti-mouse IgG or goat anti-Rabbit IgG conjugated to horseradish peroxidase (HRP) (Santa Cruz) and by enhanced chemiluminescence (Amersham).

\section{Sample preparation for PALM imaging}

The homotrimeric SNAP-Flag-TNC-TNFa was labelled with a 2-fold excess of Alexa Fluor-647 benzylguanine (NEB) in PBS (note that the homotrimer contains three SNAP subunits, which ensures efficient labeling of the homotrimer with at least one fluorophore). For cell seeding, 8well flexiPERM reusable slides (Sarstedt) mounted on glass slides (Thermo Scientific) were washed in isopropyl alcohol (Merck) for $15 \mathrm{~min}$ and plasma-cleaned in $\mathrm{N}_{2}$ for $15 \mathrm{~min}$ (Diener Electronic) to reduce background. The cleaned slides were coated for 90 min with $0.8 \mathrm{mg} / \mathrm{ml}$ Poly-L-Lysine (PLL) (Merck) coupled to polyethylene glycol (PEG) (Rapp Polymere) and a peptide containing the RGD motif (PLL-PEG-RGD) to facilitate cell adhesion. For TNFR1- 
mEos2 SMLM, $1.5 \times 10^{4}$ TNFR1/2- MEFs stably reconstituted with TNFR1-mEos2 WT, TNFR1 K32A or TNFR1 N66F were seeded in $300 \mu \mathrm{l} /$ well serum-free RPMI Medium (Thermo Scientific), $1 \%$ glutamax (Thermo Scientific), $100 \mathrm{U} / \mathrm{ml}$ penicillin (Thermo Scientific), $100 \mu \mathrm{g} / \mathrm{ml}$ streptomycin (Thermo Scientific) and $50 \mu \mathrm{g} / \mathrm{ml}$ gentamycin (Thermo Scientific). After $24 \mathrm{~h}$ of starvation, seeded cells were induced with $100 \mathrm{ng} / \mathrm{ml}(2.4 \mathrm{nM})$ SNAP-Flag-TNC-TNFa labelled with Alexa Fluor 647 in ice-cold serum-free medium at $4{ }^{\circ} \mathrm{C}$ for $30 \mathrm{~min}$. Cells were washed three times with $300 \mu \mathrm{l}$ of $400 \mathrm{mM}$ sucrose (Sigma), dissolved in sterile filtered phosphate buffered saline (PBS) (Thermo Scientific) and fixed in $300 \mu 4 \%$ formaldehyde (Thermo Scientific, ), $0.1 \%$ glutaraldehyde (Merck) and $400 \mathrm{mM}$ sucrose in sterile filtered PBS. After fixation, cells were washed three times with sterile filtered PBS.

\section{Single-molecule localization microscopy (SMLM)}

SMLM imaging (PALM) was performed on a custom-built microscope that was essentially described previously (27). The microscope is equipped with three laser modules emitting at $638 \mathrm{~nm}$ (LBX-638-180, Oxxius, $4.6 \mathrm{Wcm}^{-2}$ ), $568 \mathrm{~nm}$ (Sapphire $568 \mathrm{LP}$, Coherent; $0.21 \mathrm{kWcm}$ 2) and $405 \mathrm{~nm}$ (LBX-405-50-CSB-PP, Oxxius; 0-8.0 $\left.\mathrm{mWcm}^{-2}\right)$. The laser lines were combined with appropriate dichroic mirrors (AHF) and directed onto a $100 \mathrm{x}$ oil immersion objective (PLAPO 100x TIRFM, NA $\geq 1.45$, Olympus) mounted on an inverted microscope (IX71, Olympus). A movable mirror enabled repositioning of the laser beam to achieve total internal reflection fluorescence (TIRF). Fluorescence light that had passed through the appropriate filters (ET 700/75 for Alexa Fluor 647, Brightline HC 590/20 for mEos2; AHF) was detected with an EMCCD camera (iXon Ultra (X-10971), Andor), operated at an integration time of 100 ms, a pre-amplifier gain of 1 , and an electron multiplying gain of 200 . Single-molecule movies were recorded between 50,000 and 100,000 frames until no further blinking events were detected. An average localization precision of $14+/-1.4 \mathrm{~nm}$ was determined from a representative set of 62 images using a nearest-neighbor approach (40). The cell surface of 100 TNFR1/2- + TNFR1-mEos2 cells was determined from bright light images and quantitative analysis with Fiji (41).

\section{Quantitative image analysis}

Superresolved images were generated from PALM data using rapidSTORM (42) and postprocessed with LAMA (43). Quantitative analysis of TNFR1 clustering was performed with a method developed and applied previously $(27,29,31)$. Briefly, the number of emission events was determined for each cluster in the superresolved image (Fig. S3A), applying a threshold 
of 63 photons. Photon numbers per single-molecule detection event were obtained by converting electron counts of the EMCCD with a camera-specific correction factor. Signals from mEos2, which appeared in consecutive camera frames within a radius of $90 \mathrm{~nm}$, were grouped together as a single localization by a Kalman-filtering algorithm implemented in rapidSTORM. For the determination of the oligomeric state, the number of blinking events of $>500$ single superresolved clusters per condition were plotted and fitted with appropriate functions as described previously (31). Clusters with low brightness, a diameter $>150 \mathrm{~nm}$, low circularity, or in close vicinity to neighboring clusters (distance of $<100 \mathrm{~nm}$ ) were discarded from the analysis. For each experimental condition, at least 10 cells from at least three independent experiments were analyzed. Experimental PALM data (Figs. 2C, 3D, 4A, 4B, 4C and 4D) were fit with all possible combinations of model functions, including monomer, dimer, and trimer (31), resulting in seven different possibilities to analyze the data. These were three possibilities for a single component (monomer, dimer and trimer); three possibilities for two components (monomer/dimer, monomer/trimer and dimer/trimer); and one possibility for three components (monomer/dimer/trimer). The fit quality and the appropriateness of the number of free parameters were assessed using the Bayesian information criterion (BIC). For an in-depth discussion of the application of the BIC in the quantification of protein oligomers in mixed populations, refer to Hummer et al. (31).

\section{Supplementary Materials (see "Supplementary Materials list" below)}

Fig. S1. TNFR1 immunoprecipitations with Flag-tagged TNFa reveal the predominant ligandinteracting TNFR1 isoforms.

Fig. S2. Analysis of TNFa/TNFR1-mEos2 binding in MEFs by flow cytometry and TNFa binding assays.

Fig. S3. The concept of quantitative single-molecule localization microscopy.

Fig. S4. Functional characterization of SNAP-Flag-TNC-TNFa.

Table S1. Statistical analysis of qPALM data for unliganded WT TNFR1.

Table S2. Statistical analysis of qPALM data for stimulated WT TNFR1 that colocalized with TNF $\alpha$.

Table S3. Statistical analysis of qPALM data for stimulated WT TNFR1 that did not colocalize with TNF $\alpha$. 
Table S4. Statistical analysis of qPALM data for unliganded TNFR1 K32A.

Table S5. Statistical analysis of qPALM data for stimulated TNFR1 K32A.

Table S6. Statistical analysis of qPALM data for unliganded TNFR1 N66F.

Table S7. Statistical analysis of qPALM data for stimulated TNFR1 N66F.

\section{References and Notes}

1. P. W. Dempsey, S. E. Doyle, J. Q. He, G. Cheng, The signaling adaptors and pathways activated by TNF superfamily. Cytokine \& Growth Factor Reviews 14, 193-209 (2003)10.1016/s 1359-6101(03)00021-2).

2. B. B. Aggarwal, Signalling pathways of the TNF superfamily: A double-edged sword. Nature reviews. Immunology 3, 745-756 (2003)10.1038/nri1184).

3. R. M. Locksley, N. Killeen, M. J. Lenardo, The TNF and TNF receptor superfamilies: integrating mammalian biology. Cell 104, 487-501 (2001); published online EpubFeb 23(

4. A. Annibaldi, P. Meier, Checkpoints in TNF-Induced Cell Death: Implications in Inflammation and Cancer. Trends in molecular medicine 24, 49-65 (2018); published online EpubJan (10.1016/j.molmed.2017.11.002).

5. H. Wajant, P. Scheurich, TNFR1-induced activation of the classical NF-kappaB pathway. FEBS J 278, 862-876 (2011); published online EpubApr (10.1111/j.17424658.2011.08015.x).

6. M. A. Rivas, R. P. Carnevale, C. J. Proietti, C. Rosemblit, W. Beguelin, M. Salatino, E. H. Charreau, I. Frahm, S. Sapia, P. Brouckaert, P. V. Elizalde, R. Schillaci, TNF alpha acting on TNFR1 promotes breast cancer growth via p42/P44 MAPK, JNK, Akt and NF-kappa B-dependent pathways. Experimental cell research 314, 509-529 (2008); published online EpubFeb 1 (10.1016/j.yexcr.2007.10.005).

7. L. M. Sedger, M. F. McDermott, TNF and TNF-receptors: From mediators of cell death and inflammation to therapeutic giants - past, present and future. Cytokine Growth Factor Rev 25, 453-472 (2014); published online EpubAug (10.1016/j.cytogfr.2014.07.016).

8. S. L. Petersen, M. Peyton, J. D. Minna, X. Wang, Overcoming cancer cell resistance to Smac mimetic induced apoptosis by modulating cIAP-2 expression. Proceedings of the National Academy of Sciences of the United States of America 107, 11936-11941 (2010); published online EpubJun 29 (10.1073/pnas.1005667107).

9. N. Holler, R. Zaru, O. Micheau, M. Thome, A. Attinger, S. Valitutti, J. L. Bodmer, P. Schneider, B. Seed, J. Tschopp, Fas triggers an alternative, caspase-8-independent cell death pathway using the kinase RIP as effector molecule. Nature immunology 1, 489495 (2000); published online EpubDec (10.1038/82732).

10. A. T. Ting, M. J. M. Bertrand, More to Life than NF-kappaB in TNFR1 Signaling. Trends in immunology 37, 535-545 (2016); published online EpubAug (10.1016/j.it.2016.06.002).

11. N. Segueni, S. Benmerzoug, S. Rose, A. Gauthier, M. L. Bourigault, F. Reverchon, A. Philippeau, F. Erard, M. Le Bert, H. Bouscayrol, T. Wachter, I. Garcia, G. Kollias, M. Jacobs, B. Ryffel, V. F. Quesniaux, Innate myeloid cell TNFR1 mediates first line defence against primary Mycobacterium tuberculosis infection. Scientific reports 6, 22454 (2016); published online EpubMar 2 (10.1038/srep22454). 
12. M. M. Rahman, G. McFadden, Modulation of tumor necrosis factor by microbial pathogens. PLoS pathogens 2, e4 (2006); published online EpubFeb (10.1371/journal.ppat.0020004).

13. D. S. Vinay, B. S. Kwon, The tumour necrosis factor/TNF receptor superfamily: therapeutic targets in autoimmune diseases. Clinical and experimental immunology 164, 145-157 (2011); published online EpubMay (10.1111/j.1365-2249.2011.04375.x).

14. I. Martinez-Reza, L. Diaz, R. Garcia-Becerra, Preclinical and clinical aspects of TNFalpha and its receptors TNFR1 and TNFR2 in breast cancer. Journal of biomedical science 24, 90 (2017); published online EpubDec 4 (10.1186/s12929-017-0398-9).

15. D. W. Banner, A. D'Arcy, W. Janes, R. Gentz, H.-J. Schoenfeld, C. Broger, H. Loetscher, W. Lesslauer, Crystal structure of the soluble human $55 \mathrm{kd}$ TNF receptorhuman TNF $\beta$ complex: Implications for TNF receptor activation. Cell 73, 431-445 (1993)10.1016/0092-8674(93)90132-a).

16. F. K.-M. Chan, Three is better than one: Pre-ligand receptor assembly in the regulation of TNF receptor signaling. Cytokine 37, 101-107 (2007)10.1016/j.cyto.2007.03.005).

17. F. K. Chan, H. J. Chun, L. Zheng, R. M. Siegel, K. L. Bui, M. J. Lenardo, A domain in TNF receptors that mediates ligand-independent receptor assembly and signaling. Science (New York, N.Y.) 288, 2351-2354 (2000).

18. M. Branschadel, A. Aird, A. Zappe, C. Tietz, A. Krippner-Heidenreich, P. Scheurich, Dual function of cysteine rich domain (CRD) 1 of TNF receptor type 1: conformational stabilization of CRD2 and control of receptor responsiveness. Cell Signal 22, 404-414 (2010); published online EpubMar (10.1016/j.cellsig.2009.10.011).

19. J. Cao, F. Meng, X. Gao, H. Dong, W. Yao, Expression and purification of a natural Nterminal pre-ligand assembly domain of tumor necrosis factor receptor 1 (TNFR1 PLAD) and preliminary activity determination. The protein journal 30, 281-289 (2011); published online EpubApr (10.1007/s10930-011-9330-4).

20. H. Wajant, Principles of antibody-mediated TNF receptor activation. Cell death and differentiation 22, 1727-1741 (2015); published online EpubNov (10.1038/cdd.2015.109).

21. V. Boschert, A. Krippner-Heidenreich, M. Branschädel, J. Tepperink, A. Aird, P. Scheurich, Single chain TNF derivatives with individually mutated receptor binding sites reveal differential stoichiometry of ligand receptor complex formation for TNFR1 and TNFR2. Cellular signalling 22, 1088-1096 (2010)10.1016/j.cellsig.2010.02.011).

22. J.-L. Bodmer, P. Schneider, J. Tschopp, The molecular architecture of the TNF superfamily. Trends in biochemical sciences 27, 19-26 (2002).

23. J. H. Naismith, T. Q. Devine, B. J. Brandhuber, S. R. Sprang, Crystallographic evidence for dimerization of unliganded tumor necrosis factor receptor. The Journal of biological chemistry 270, 13303-13307 (1995); published online EpubJun 2 (

24. M. Sauer, M. Heilemann, Single-Molecule Localization Microscopy in Eukaryotes. Chemical reviews 117, 7478-7509 (2017); published online EpubJun 14 (10.1021/acs.chemrev.6b00667).

25. A. Fürstenberg, M. Heilemann, Single-molecule localization microscopy-nearmolecular spatial resolution in light microscopy with photoswitchable fluorophores. Physical chemistry chemical physics : PCCP 15, 14919-14930 (2013)10.1039/c3cp52289j).

26. S. H. Lee, J. Y. Shin, A. Lee, C. Bustamante, Counting single photoactivatable fluorescent molecules by photoactivated localization microscopy (PALM). Proceedings of the National Academy of Sciences of the United States of America 109, 17436-17441 (2012); published online EpubOct 23 (10.1073/pnas.1215175109). 
27. F. Fricke, J. Beaudouin, R. Eils, M. Heilemann, One, two or three? Probing the stoichiometry of membrane proteins by single-molecule localization microscopy. Scientific reports 5, 14072 (2015)10.1038/srep14072).

28. R. Jungmann, M. S. Avendano, M. Dai, J. B. Woehrstein, S. S. Agasti, Z. Feiger, A. Rodal, P. Yin, Quantitative super-resolution imaging with qPAINT. Nature methods 13, 439-442 (2016); published online EpubMay (10.1038/nmeth.3804).

29. C. L. Krüger, M.-T. Zeuner, G. S. Cottrell, D. Widera, M. Heilemann, Quantitative single-molecule imaging of TLR4 reveals ligand-specific receptor dimerization. Science signaling 10, (2017)10.1126/scisignal.aan1308).

30. E. Betzig, G. H. Patterson, R. Sougrat, O. W. Lindwasser, S. Olenych, J. S. Bonifacino, M. W. Davidson, J. Lippincott-Schwartz, H. F. Hess, Imaging intracellular fluorescent proteins at nanometer resolution. Science (New York, N.Y.) 313, 1642-1645 (2006)10.1126/science.1127344).

31. G. Hummer, F. Fricke, M. Heilemann, Model-independent counting of molecules in single-molecule localization microscopy. Molecular biology of the cell 27, 3637-3644 (2016)10.1091/mbc.E16-07-0525).

32. F. Fricke, J. Beaudouin, R. Eils, M. Heilemann, One, two or three? Probing the stoichiometry of membrane proteins by single-molecule localization microscopy. Scientific reports 5, 14072 (2015); published online EpubSep 11 (10.1038/srep14072).

33. H. Wajant, D. Siegmund, TNFR1 and TNFR2 in the Control of the Life and Death Balance of Macrophages. Front Cell Dev Biol 7, 91 (2019)10.3389/fcell.2019.00091).

34. H. Tomoda, Y. Kishimoto, Y. C. Lee, Temperature effect on endocytosis and exocytosis by rabbit alveolar macrophages. The Journal of biological chemistry 264, 15445-15450 (1989); published online EpubSep 15 (

35. H. H. Jarosz-Griffiths, J. Holbrook, S. Lara-Reyna, M. F. McDermott, TNF receptor signalling in autoinflammatory diseases. International immunology, (2019); published online EpubMar 6 (10.1093/intimm/dxz024).

36. A. Krippner-Heidenreich, F. Tubing, S. Bryde, S. Willi, G. Zimmermann, P. Scheurich, Control of receptor-induced signaling complex formation by the kinetics of ligand/receptor interaction. The Journal of biological chemistry 277, 44155-44163 (2002); published online EpubNov 15 (10.1074/jbc.M207399200).

37. M. L. Gaeta, D. R. Johnson, M. S. Kluger, J. S. Pober, The death domain of tumor necrosis factor receptor 1 is necessary but not sufficient for Golgi retention of the receptor and mediates receptor desensitization. Laboratory investigation; a journal of technical methods and pathology 80, 1185-1194 (2000); published online EpubAug (

38. H. Storey, A. Stewart, P. Vandenabeele, J. P. Luzio, The p55 tumour necrosis factor receptor TNFR1 contains a trans-Golgi network localization signal in the C-terminal region of its cytoplasmic tail. The Biochemical journal 366, 15-22 (2002); published online EpubAug 15 (10.1042/BJ20020048).

39. F. Fricke, S. Malkusch, G. Wangorsch, J. F. Greiner, B. Kaltschmidt, C. Kaltschmidt, D. Widera, T. Dandekar, M. Heilemann, Quantitative single-molecule localization microscopy combined with rule-based modeling reveals ligand-induced TNF-R1 reorganization toward higher-order oligomers. Histochemistry and cell biology 142, 91101 (2014); published online EpubJul (10.1007/s00418-014-1195-0).

40. U. Endesfelder, S. Malkusch, F. Fricke, M. Heilemann, A simple method to estimate the average localization precision of a single-molecule localization microscopy experiment. Histochemistry and cell biology 141, 629-638 (2014); published online EpubJun (10.1007/s00418-014-1192-3).

41. J. Schindelin, I. Arganda-Carreras, E. Frise, V. Kaynig, M. Longair, T. Pietzsch, S. Preibisch, C. Rueden, S. Saalfeld, B. Schmid, J. Y. Tinevez, D. J. White, V. Hartenstein, K. Eliceiri, P. Tomancak, A. Cardona, Fiji: an open-source platform for biological- 
image analysis. Nature methods 9, 676-682 (2012); published online EpubJun 28 (10.1038/nmeth.2019).

42. S. Wolter, M. Schuttpelz, M. Tscherepanow, V. D. L. S, M. Heilemann, M. Sauer, Realtime computation of subdiffraction-resolution fluorescence images. Journal of microscopy 237, 12-22 (2010); published online EpubJan (10.1111/j.13652818.2009.03287.x).

43. S. Malkusch, M. Heilemann, Extracting quantitative information from single-molecule super-resolution imaging data with LAMA - LocAlization Microscopy Analyzer. Scientific reports 6, 34486 (2016)10.1038/srep34486).

Acknowledgments: The authors acknowledge Daniela Männel (Regensburg) for $T N F R 1 / 2^{-/-}$ MEFs and Domagoj Vuvic (Genentech Inc.) for providing BV6.

Funding: This work was supported by the German Science Foundation (grants SFB 807, HE 6166/11-1, and EXC115 to C. K. and M. H.; and SFB 1177 and EXC 115 to I. D.) and the Bundesministerium für Bildung und Forschung (BMBF:eBio to S. M. and M. H.). S. J. L. v. W. acknowledges funding of the German Science Foundation (WI 5171/1-1) and the Dr. Eberhard and Hilde Rüdiger Foundation. S. F. acknowledges funding from the LOEWE initiative (Dynamem). H. W. acknowledges funding by the DFG (grant WA 1025/24-1).

Author contributions: S. J. L. v. W., I. D. and M. H. designed the study. C. K., J. M., S. J. L. v. W., and F. F. performed experiments, and together with S. M., D. W., M. H. and H. W., analyzed the data. S. J. L. v. W., C. K. and M. H. wrote the manuscript. All authors discussed and edited the manuscript. Competing interests: The authors declare that they have no competing interests. Data and materials availability: All data needed to evaluate the conclusions in the paper are present in the paper or the Supplementary Materials.

\section{Figure Legends}

Fig. 1. Stable reconstitution of TNFR1/2/- MEFs with TNFR1-mEos2 rescues TNF $\alpha-$ dependent and TNFR-mediated NF-kB signaling. (A) Cell lysates of $T N F R 1 / 2^{+/+}$and TNFR1/2/- MEFs alone or reconstituted with TNFR1-mEos2 were analyzed by Western blotting with antibodies against TNFR1 and vinculin as a loading control. Asterisks indicate nonspecific bands. HeLa cell extracts were analyzed as positive controls. Western blots are representative of three independent experiments. (B) TNF $\alpha$-induced TNFR1 activation at the indicated times in the indicated MEFs was evaluated by Western blotting analysis of the phosphorylation and degradation of $\mathrm{I} \mathrm{KB} \alpha$. Vinculin was used as a loading control. Western 
blots are representative of three independent experiments. (C) TNF $\alpha$-induced nuclear translocation of p65in the indicated MEFs was analyzed by immunofluorescence staining of p65. DNA was counterstained by DAPI. Right: Panels show the mEos2 fluorescence signal. Scale bars: $10 \mu \mathrm{m}$. Images are representative of three independent experiments. (D) Evaluation of TNFa-induced programmed cell death. The indicated MEFs were left untreated (-) or were treated with TNF $(10 \mathrm{ng} / \mathrm{ml})(\mathrm{T})$ and $20 \mu \mathrm{M}$ zVAD.fmk (Z) for 48 hours after 30-min pretreatment with $10 \mu \mathrm{M}$ BV6 (B). The percentage of propidium iodide (PI)-positive cells of the total cell number was quantified as a measure for cell death. EV, empty vector. Data are means $\pm \mathrm{SD}$ of at least three experiments performed in triplicate. ${ }^{* *} P<0.001$.

Fig. 2. Quantitative superresolution microscopy reveals the oligomeric state of unliganded TNFR1 in TNFR1/2/ + TNFR1-mEos2 MEFs. (A) PALM image of unliganded TNFR1/2- + TNFR1-mEos2 MEFs. Image is representative of 10 cells in three independent experiments. (B) Magnified view of the boxed region in (A). (C) The oligomeric state was determined by analyzing the single-molecule blinking signatures of mEos2 (histogram). PDB: 1NCF, 1TNF. Scale bars: $5 \mu \mathrm{m}$ (bright light), $2 \mu \mathrm{m}(\mathrm{A}), 1 \mu \mathrm{m}(\mathrm{B})$.

Fig. 3. Quantitative superresolution microscopy reveals the oligomeric state of activated TNFR1 in TNFR1/2/- TNFR1-mEos2. (A) PALM image of a TNFR1/2/- + TNFR1mEos2 cell with TNF $\alpha$-activated TNFR1 (TNF $\alpha$-SNAP-Alexa Fluor 647). Image is representative of 14 cells in three independent experiments. (B) Magnified view of the boxed region in (A), indicating ligand-free TNFR1 (green circles) and ligand-bound TNFR1 (orange circles). (C) The oligomeric states of ligand-bound TNFR1 (orange circles in (B)) were determined by analyzing single-molecule blinking signatures of mEos2 (histogram). PDB: 1NCF, 1TNF. (D) The oligomeric state of ligand-free TNFR1 (green circles in (B)) was determined by analyzing single-molecule blinking signatures of mEos2. Scale bars: $5 \mu \mathrm{m}$ (bright light), $2 \mu \mathrm{m}(\mathrm{A}), 1 \mu \mathrm{m}(\mathrm{B})$.

Fig. 4. Quantitative superresolution microscopy of TNFR1 carrying mutations in the PLAD or ligand-binding domain in TNFR1/2- + TNFR1-mEos2 MEFs. (A and B) PALM images of $T N F R 1 / 2^{-\alpha}+$ TNFR1-mEos2 MEFs with TNFR1 carrying a mutation in the PLAD (K32A), untreated cells $(\mathbf{A})$ and cells treated with TNF $\alpha(B)$. Images are representative of 10 cells in three independent experiments. The oligomeric states of TNFR1 K32A mutant were determined by analyzing single-molecule blinking signatures of mEos2 (histogram). (C and $\mathbf{D})$ PALM images of TNFR1/2/ + TNFR1-mEos2 MEFs with TNFR1 carrying a mutation in the ligand-binding domain (N66F), either untreated (C) or treated with TNF $\alpha$ (D). Images are representative of 10 cells in three independent experiments. The oligomeric state of TNFR1 N66F mutant were determined by analyzing single-molecule blinking signatures of mEos2 
(histogram). PDB: 1NCF. Scale bars: $5 \mu \mathrm{m}$ (bright light), $2 \mu \mathrm{m}$ (A-D). (E) Western blot analysis of total cell lysates of the indicated TNFR1/2 ${ }^{-\alpha}+$ TNFR1-mEos2 MEFs, probed with antiTNFR1. Vinculin was used as a loading control. Asterisks indicate background bands. Western blots are representative of three independent experiments. (F) Evaluation of NF-KB functionality in the indicated MEFs by assessing TNFa-induced IKB $\alpha$ phosphorylation and degradation. Western blots are representative of three independent experiments. Vinculin was used as a loading control

Fig. 5. A molecular model for TNFR1 activation in $T N F R 1 / 2^{--}+$TNFR1-mEos2. Unliganded TNFR1 resides in an equilibrium of monomeric and dimeric receptors, with the PLAD as the determinant for dimerization. The presence of dimers is crucial for TNF $\alpha$-induced activation of TNFR1, which occurs through the formation of trimeric and higher orders of TNFR1. Mutations in the PLAD prevents the formation of dimers (PDB: 1NCF, 1TNF).

Table 1. Cluster per $\mu \mathrm{m}^{2}$ and radii determined by PALM. For WT TNFR1, TNFR1 K32A and TNFR1 N66F, the number of cluster per $\mu \mathrm{m}^{2}$ and the radii are given.

\begin{tabular}{l|ll}
\multicolumn{1}{l}{ Condition } & Cluster/ $\mathbf{\mu m}^{2}$ & Radius [nm] \\
\hline TNFR-mEos2 WT & $1.4+/-0.4$ & $17+/-13$ \\
- TNF $\alpha$ & $1.6+/-0.7$ & $19+/-4$ \\
+ TNF $\alpha$ & $0.2+/-0.1$ & $16+/-10$ \\
\hline TNFR1-mEos2 K32A & $15+/-5$ \\
-TNF $\alpha$ & $0.3+/-0.2$ & \\
+ TNF $\alpha$ & $0.5+/-0.2$ & $16+/-9$ \\
\hline TNFR1-mEos2 N66F & $0.6+/-0.4$ & $17+/-10$ \\
-TNF $\alpha$ &
\end{tabular}

Table 2. Quantitative analysis of TNFR1 clustering as determined by PALM. The oligomeric state of receptor clusters $(M=$ monomer, $D=$ dimer, $T=$ trimer) is given for $\mathrm{WT}$ TNFR1-mEos2, TNFR1-mEos2 K32A and TNFR1-mEos2 N66F.

Condition Oligomeric state TNFR1-mEos2 WT $-\mathrm{TNF} \alpha$
$66+/-4 \%(\mathrm{M}), 34+/-4 \%(\mathrm{D})$ 


\begin{tabular}{l|l}
$\begin{array}{l}\text { +TNF } \alpha \text { (only TNFR1 clusters co localizing with } \\
\text { TNF } \alpha \text { were analysed) }\end{array}$ & $13+/-2 \%$ (M), $64+/-2 \%$ (T), 23+/- $3 \%$ (9mer) \\
+TNF $\alpha$ (only TNFR1 clusters not co localizing & $41+/-4 \%$ (M), $59+/-4 \%$ (D) \\
with TNF $\alpha$ were analysed) & $100+/-3 \%$ (M) \\
\hline TNFR1-mEos2 K32A & $100+/-3 \%$ (M) \\
-TNF $\alpha$ & $54+/-3 \%$ (M), $46+/-3 \%$ (D) \\
+ +TNF $\alpha$ (all TNFR1 clusters analysed) & $56+/-3 \%$ (M), $44+/-3 \%$ (D) \\
\hline TNFR1-mEos2 N66F &
\end{tabular}


Figure 1
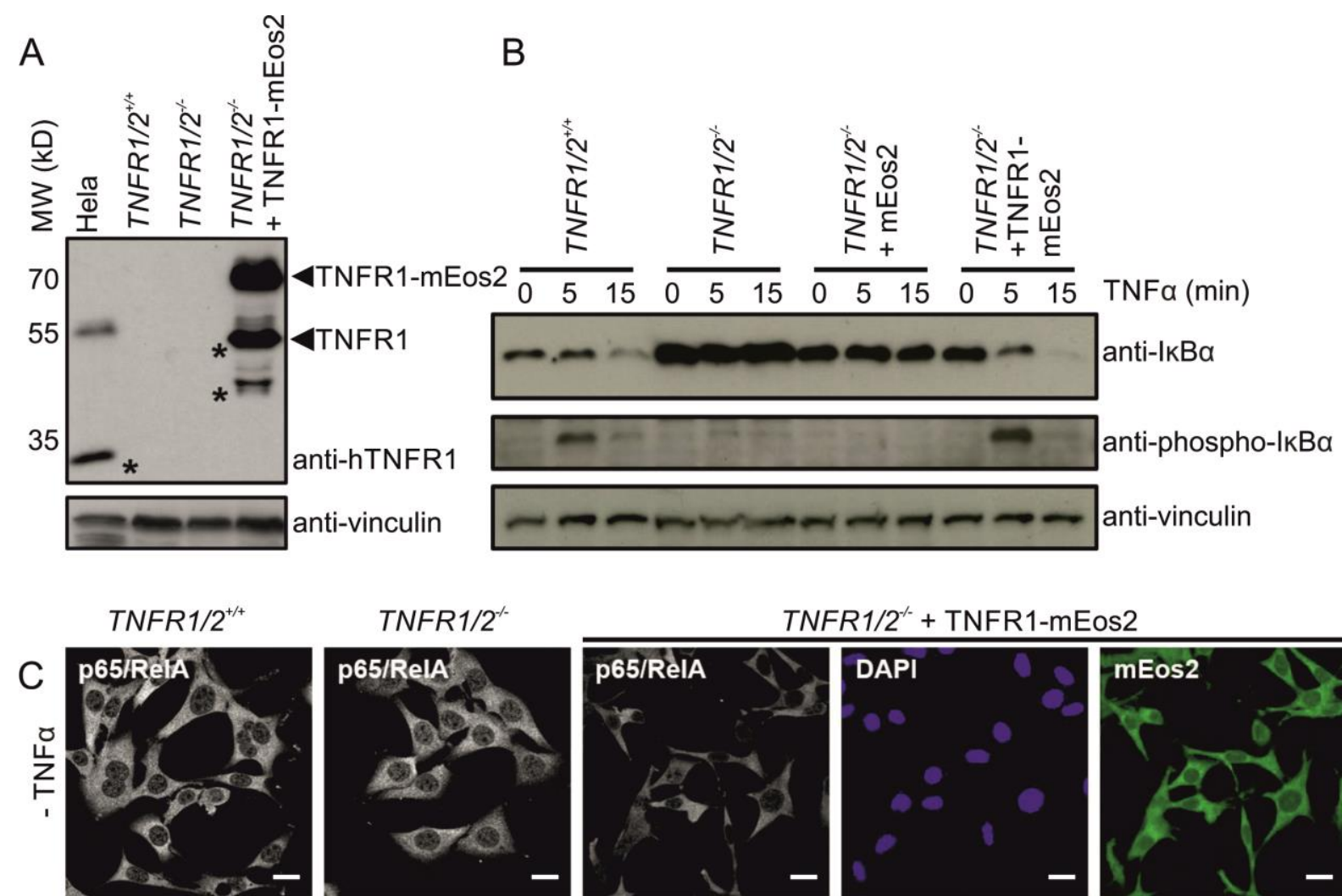

TNFR $1 / 2^{-1-}$

TNFR1/2 ${ }^{-1}$ + TNFR1-mEos2
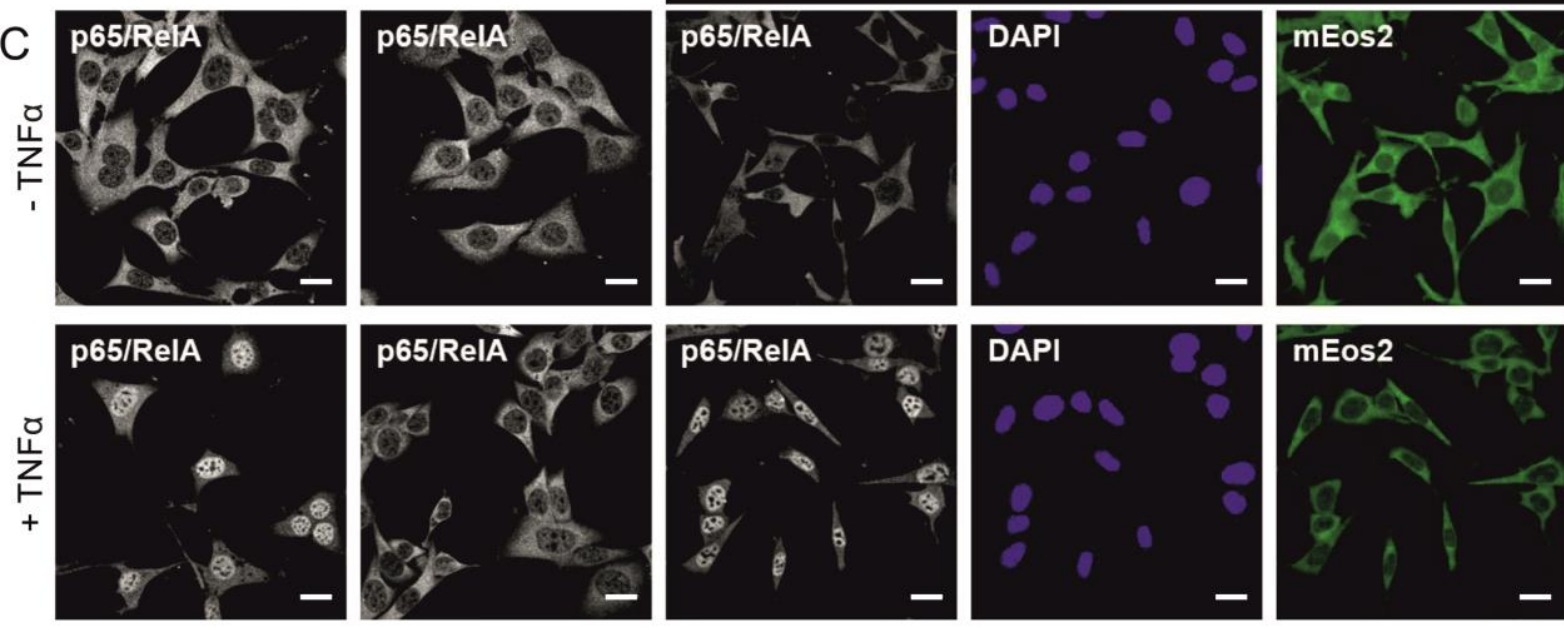

D
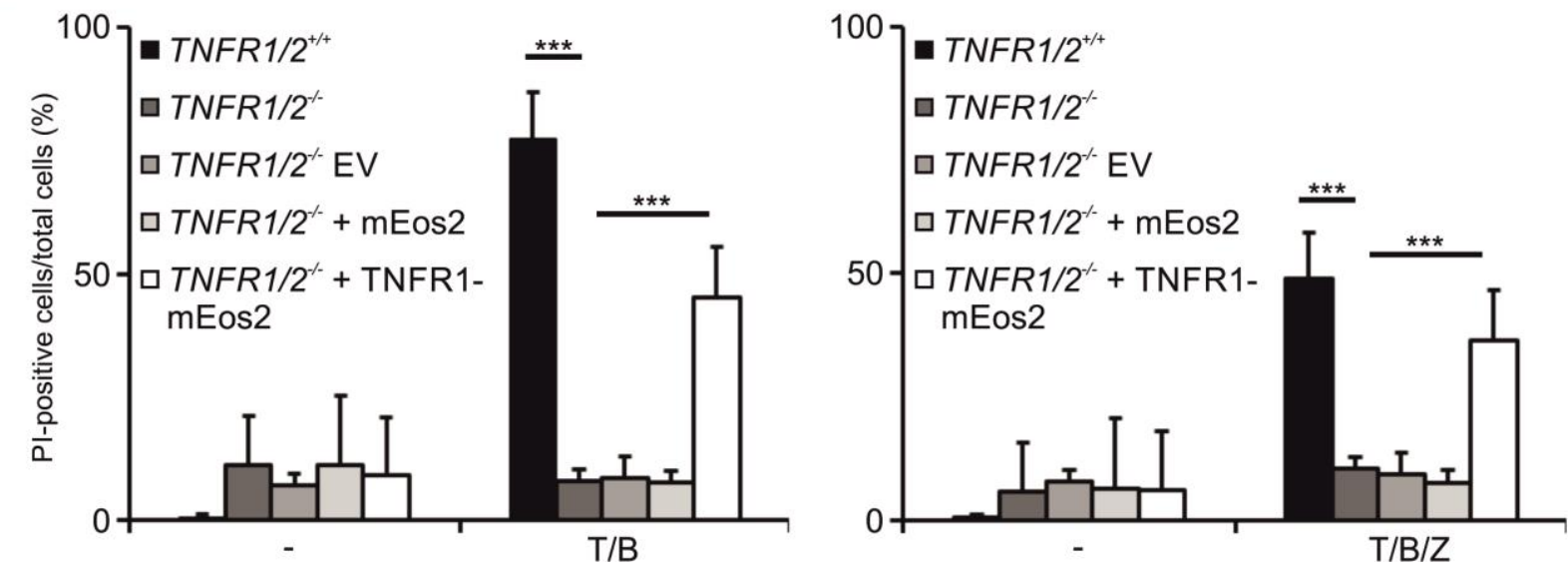
Figure 2
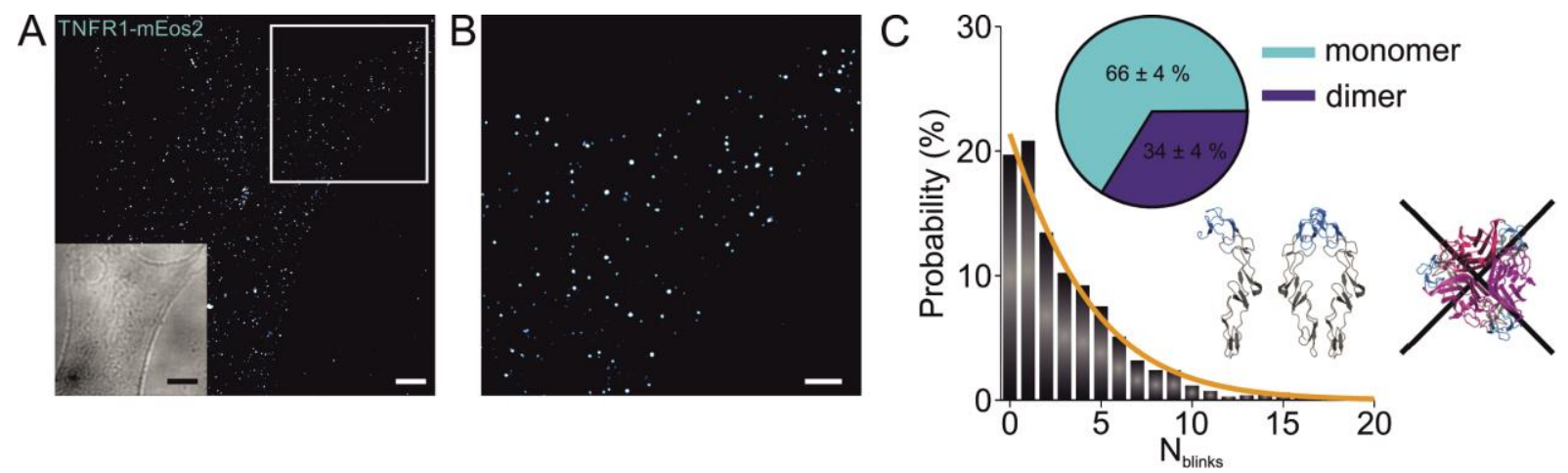
Figure 3
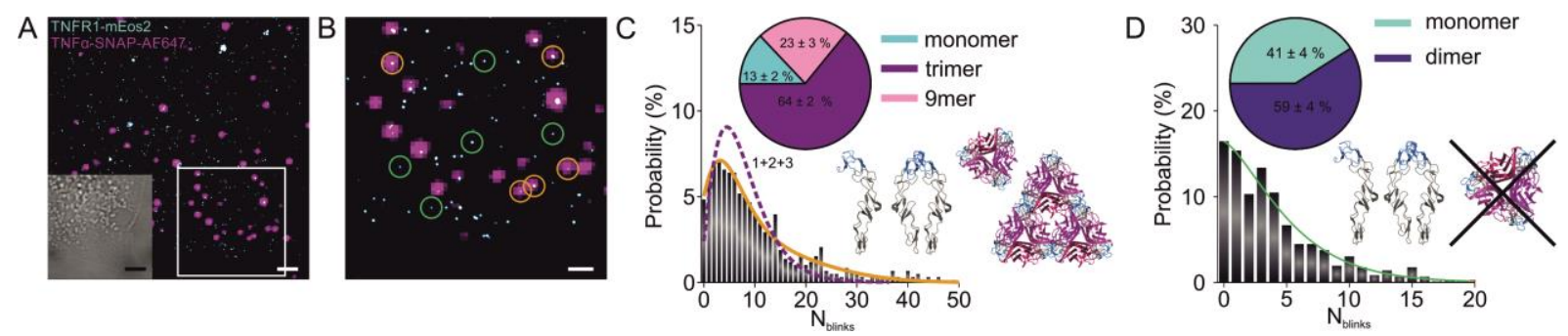
Figure 4
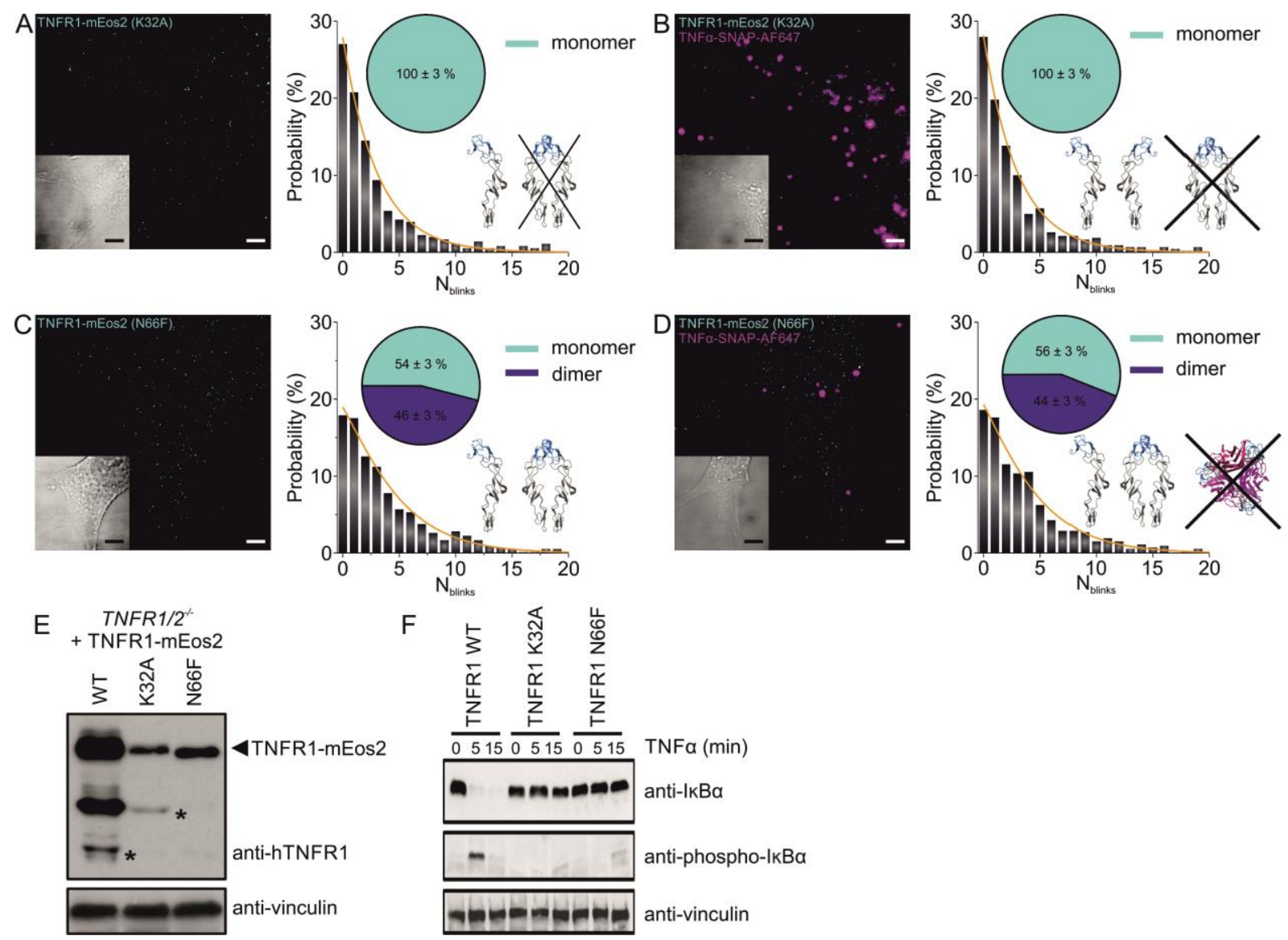
Figure 5

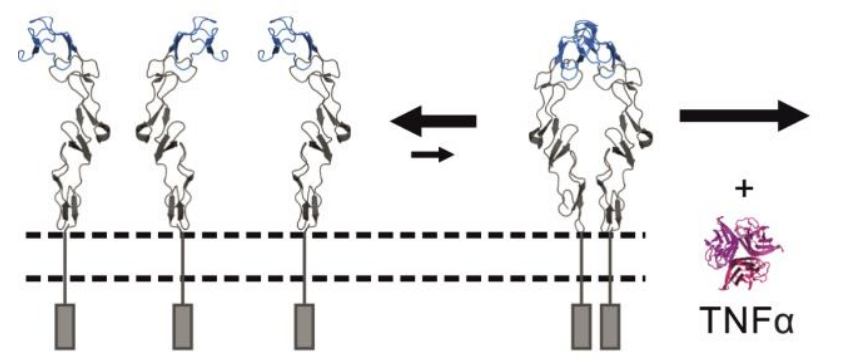

side view

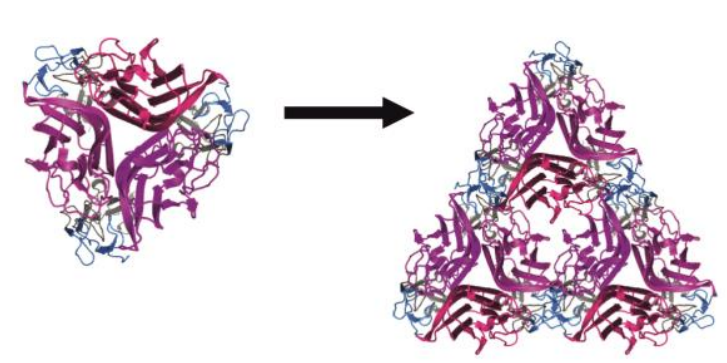

top view 